\title{
Hybrid Dynamic Simulation of Rigid-Body Contact with Coulomb Friction*
}

\author{
Wookho Son Jeffrey C. Trinkle Nancy M. Amato \\ \{wooks@cs.tamu.edu, jctrink@sandia.gov, amato@cs.tamu.edu\} \\ Departrnent of Computer Science, Texas A\&M University \\ College Station, TX 77843-3112
}

\begin{abstract}
This paper introduces a hybrid scheme for simulating rigid bodies in contact. We use an adaptive strategy for handling two different contact situations, 'bouncing' and 'steady'. To handle contact for rigid bodies, we use two impulse-based methods to explicitiy or implicitly compute impulses due to collision impact. These two methods are used so that different impulse methods are applied adaptively depending on the contact situations. Our experiments show that our simple adaptive simulation scheme enables efficient and physically-correct dynamic simulation involving rigid-body contacts with Coulomb friction. This adaptive scheme was incorporated into our dynamic simulator, called I-GMS, which supports various types of simulations. We demonstrate the simulation results of our scheme using a ball falling on a flat surface in three dimensions.
\end{abstract}

\section{Introduction}

This work is concerned with simulation of rigid body dynamics in contact with Coulumb friction. Rigid body contact problems occur in many engineering applications in which deformations due to contacts could be ignored. Also, it is gaining increasing attention from the VR, graphics, and robotics communities, since most existing simulators do not handle well the situations which involve many contact interactions due to frequent loss and gain of multiple contacts between the objects.

In previous work, Baraff [2] and Lötstedt [3] apply complementarity theory using a pivoting method such as Lemke's algorithm. Trinkle et al. [13] takes an instantaneous point of view and uses Linear Complementary Problem (LCP) formulation to compute the forces and accelerations. However, these instantaneous formulae do not always have solutions. This

*This research supported in part by NSF CAREER Award CCR-9624315, NSF Grants IIS-9619850, EIA-9805823, and EIA-9810937, and by the Texas Higher Education Coordinating Board under grant ARP-036327-017. is because the use of Coulomb's friction law with the principles of classical rigid bocty dynamics introduces mathematical inconsistencies.

To overcome these limitations, Song et al. [9] used a compliant contact model to derive stability criteria that yield a unique solution in. terms of accelerations and forces for planar systems. While it is possible to model elastic bodies as a system of springs and masses, the level of detail in the model and the stiffness of the involved differential equations makes this approach computationally expensive. Also, while the compliant contact model overcomes some of the inconsistency problems occurring during simulation, it is appealing to use simpler and more efficient rigid body models whenever the rigid body dynamics solution is unique and stable.

Mirtich developed an impulse-based algorithm using Newton and Poisson impact hypothesis, which predicts an increase of the kinetic energy for complex collision model, and Strong's hypothesis, which conserves energy [5]. Moreau [6], Marques [4], and Stewart [11] use a method which does not determine the impulse directly. Instead, they use a velocity-based time-stepping method which employs the integrals of the forces over each time step to incorporate impulses. These velocity-based methods avoid the nonexistent solution problem that plagues acceleration-based methods.

Since no existing method deals with efficient simulation of all cases of contact situations without nonexistence solution problems, in this paper we introduce a hybrid method for simulating rigid bodies in contact with friction. Our method has the capability to adaptively change contact dynamics depending on contact situation. This hybrid scheme combines the impulse-based method by Mirtich [5] and implicit time-stepping method by Stewart $[11,12]$ to efficiently deal with two common types of contact situations such as 'bouncing' and 'steady'. This hybrid scheme has been incorporated into our dynamic simulator, I-GMS $[7][8]$, so that the appropriate method is selected adaptively during real-time simulation. 


\section{Our Approach}

Our adaptive scheme handles two different contact situations, 'bouncing contact' and 'steady contact', which commonly occur during simulation of a system of rigid-bodies. In reality, a bouncing contact will be gradually diminished to a 'steady contact' after sliding and rolling, where it is considered that no more bouncing occurs. In simulation, we consider bouncing to have ended when the bounces are so small that, numerically, the contact is steady state. Correctly tracking these changes in contact situations and applying appropriate contact dynamics is very important for physically-correct motion simulation. Unfortunately, no single existing method handles all these contact situations well.

To overcome the difficulties arising in simulating rigid bodies in contact, we have developed a hybrid method which uses two impulse-based algorithms, Mirtich's impulse-based [5] and Stewart's implicit time-stepping algorithms $[11,12]$. This adaptive simulation scheme appropriately handles the transition between contact situations by enabling adaptive application of the appropriate contact dynamics. In this way, we are able to maintain real-time capability, while at the same time achieving physically-correct motion. We do pay a cost in efficiency for the case of 'steady contact', in the presence of contact transition.

To deal with bouncing contact interactions between bodies, we have used the impulse-based approach introduced by Mirtich [5]. In this scheme, all types of contacts (colliding, rolling, sliding, and resting) are modeled as a series of collision impulses between the bodies in contact. Hence, this method is simpler and faster than the constraint-based approach where a non-penetration constraint is enforced for each contact. Mirtich's impulse-based method handles well only the case of a bouncing-ball, since the system cannot be solved beyond the finite time taken for the ball come to rest. In general, these situations do not handle the case where forces are instantaneously unbounded, but are not impulsive.

To deal with 'steady contact', we use an implicit time-stepping method for simulating systems of rigid bodies developed by Stewart and Trinkle [11]. Unlike other methods which take an instantaneous point of view [13], this relatively new method does not explicitly identify impulsive forces. Instead, the method uses the integrals of the forces over each time step, which are finite even if there are impulsive forces. However, this method handles only 'steady contact' because it impilcitly assumes a zero coefficient of restitution [11].

\section{Rigid-Body Contact Model}

In this section, we present the fundamental mathematical model for three dimensional rigid-body dynamics with Coulomb frictional contact. At each time step of the continuous-time problem, the model must be formulated and solved to determine the velocities of the bodies. The velocities are then used to update the positions. After the update, the bodies are checked for collisions, upon which an impulse-based model would be applied. Then, this process would repeat.

In this paper, we describe only the relatively new 'implicit time-stepping' algorithm by Stewart [11] which is used as a component of contact dynamics in our hybrid dynamic simulator. The basic idea of this method is as follows. At each time step, the system's motion equations are formulated as a Nonlinear Complementarity Problem (NCP) in body positions and/or velocities. This NCP can be solved as a sequence of Linear Complementarity Problems (LCPs). Then, this velocity-based method avoids the problem of solution nonexistence that plagues acceleration-based methods. Mirtich's impulse-based contact model is described in [5].

\subsection{Rigid-Body Dynamics with Contact}

The mathematical formulation for the continuous problem is based on measure differential inclusions by Moreau et al. [6]. To obtain a mathematical formulation, the derivation begins with a formulation of rigid body dynamics without contact. This is done using a Lagrangian formulation with generalized coordinates $q(t)$ and generalized velocities $v(t)$. Contact is assumed to be represented by a function $f(q)$ so that if $f(q)>0$ there is no contact. If $f(q)=0$ then there is contact, and if $f(q)<0$ then $q$ is an inadmissible configuration. The set of admissible configurations is defined by

$$
C=\{q \mid f(q) \geq 0\} \subset R^{n}
$$

where $\nabla f(q) \neq 0$ whenever a configuration $q$ lies in the boundary $\partial$ of $C$ in a generalized coordinate system, $R^{n}$.

For a contact constraint $f(q) \geq 0$, if there is no contact $(f(q(t))>0)$, then the contact forces must be zero $(\psi(t)=0)$. Also, since the contact is not adhesive, the contact force cannot be directed outwards, so $\psi(t) \geq 0$. Thus, $f(q(t)) \geq 0 \forall t$. This interpretation gives a differential version of a Nonlinear Complementarity Problem (NCP) which has the following form of Newton-Euler equations for unilateral constraints:

$$
\begin{array}{r}
M(q) \frac{d \mathbf{v}}{d t}=k(q, v)+\rho(t), \\
f(q(t)) \geq 0, \forall t, \\
\rho(t) \in \widehat{F C}(q(t)), \forall t, \\
\rho(t) f(q(t))=0, \forall t,
\end{array}
$$


Here, $M(q)$ is the positive definite and symmetric inertia matrix, $k(q, v)$ represents a pseudo force such as centripetal, coriolis, and external generalized forces, and $\rho(t)$ are the total forces, consisting of the normal contact force $\psi(t) \nabla f(q(t))$ plus the generalized friction force. This corresponds to the complementarity formulation: Given $g: R^{n} \rightarrow R^{n}$, find $z$ such that

$$
z \geq 0, g(z) \geq, z^{T} g(z)=0
$$

\subsection{Incorporation of Coulomb Friction}

When two bodies are in contact, friction may exist, in which case, there are equal and opposite tangential forces acting on the two bodies in contact. If the bodies are sliding against each other, the friction forces must oppose the slip. The magnitude of the friction forces $\left(\mathbf{c}_{\mathbf{t}}\right)$ is bounded by $\mu \mathbf{n}$ where $\mu>0$ is the coefficient of friction and $\mathbf{n}$ is the normal component of the contact force. More specifically, if there is slippage, the frictional force must have exactly this magnitude (for isotropic friction), and if there is no slippage, any equal and opposite function forces within this bound are admissible.

The Coulomb friction forces are defined in terms of a friction cone which contains the sums of the normal and frictional contact forces. This is usually given for a point $q$ on the boundary of the admissible region $C \subset R^{3}$ as the subset

$$
\widehat{F C}(q)=\left\{c_{t}+c_{n} \mathbf{n} \mid\left\|c_{t}\right\|_{2} \leq \mu c_{n}, c_{t} \perp \mathbf{n}\right\}
$$

where $\mathbf{n}$ is the normal vector of $C$ at $q$ pointing to the admissible region, $c_{n}$ and $c_{t}$ are the normal and friction forces respectively, and $\mu>0$ is the coefficient of friction.

To linearize Equation (6), we use a polyhedral approximation to the friction cone $(\widehat{F C})$ which defines the Coulomb friction forces at the contact as follows:

$$
\widehat{F C}(q)=\left\{c_{n} \mathbf{n}+D \beta \mid c_{n} \geq 0, \beta \geq 0, e^{T} \beta \leq \mu c_{n}\right\}
$$

where $e=[1,1, \ldots, 1]^{T}$ is in $K^{k}, k$ is the number of edges of the polyhedral approximation, $c_{n}$ denotes the magnitude of the normal contact force, and $\mathbf{n}$ is an untransformed normal vector at the contact. (Note that if there is only one moving body; then, $\mathbf{n}$ can be the 6 dimensional unit wrench of the normal component of the contact force.) $D$ is a matrix the columns of which are direction vectors $d_{j}$ that positively span the subspace on which the friction forces act. (It is assumed that for every $i$ there is a $j$ such that $d_{i}=-d_{j}$.) The vector $\beta$ is used as a weighting vector for the direction vectors $d_{i}$. An example of this approximation for the case $k=8$ is given in Figure 1

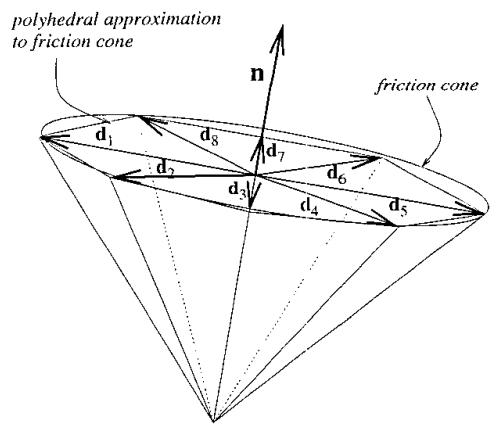

Figure 1: Polyhedral approximation of friction cone $(\mathrm{k}=8)$

\subsection{Complementarity Formulation of Time-Stepping}

We cast the Newton-Euler equation, nonpenetration constraints, and a friction law as a linear complementarity problem in a discrete-time form used in simulation. The approach taken here is to construct a time-stepping method which uses integrals of the applied forces to compute integrals of contact forces over small time intervals. The time-stepping formulation is a variant of the well-known implicit Euler method for ODE's.

The formulation of the time-stepping is then to approximate the position $q^{t+1}$ and velocity $v^{t+1}$ and the associated variables $c_{n}, \beta$, and $\lambda$ in Equation (4). This yields discretized equations:

$$
\begin{aligned}
M\left(q^{t}+h v^{t}\right) \cdot\left(v^{t+1}-v^{t}\right) & \\
\mathbf{n} c_{n}+D \beta+h k\left(q^{t}+h v^{t} / 2, v^{t}\right), & \\
q^{t+1}-q^{t} & =h v^{t+1}, \\
\lambda e+D^{T} v^{t+1} & \geq 0, \beta \geq 0, \\
\mathbf{n}^{T} q^{t+1} & \geq \alpha_{0}, c_{n} \geq 0, \\
\mu c_{n}-e^{T} \beta & \geq 0, \lambda \geq 0
\end{aligned}
$$

This yields the complementarity conditions

$$
\begin{aligned}
{\left[\lambda e+D^{T} v^{t+1}\right]^{T} \beta } & =0 \\
{\left[\mathbf{n}^{T} q^{t+1}-\alpha_{0}\right] c_{n} } & =0 \\
{\left[\mu c_{n}-e^{T} \beta\right] \lambda } & =0
\end{aligned}
$$

where the additional variable $\lambda$ is an approximation to the magnitude of the relative contact velocity, $c_{n}$ and $D \beta$ represent the impulses of the normal contact force and the friction force at the contact over the period of one time step, respectively. Here, $\lambda e+D^{T} v^{t+1} \geq 0$ constrains the friction force to the facet of the linearized friction cone, $\mathbf{n}^{T} q^{t+1} \geq \alpha_{0}$ is the approximation of the nonpenetration constraint $(f(q) \geq 0)$ by a half-space, and $\left[\mu c_{n}-e^{T} \beta\right] \lambda \geq 0$ constrains the contact force not to go outside the friction cone. 


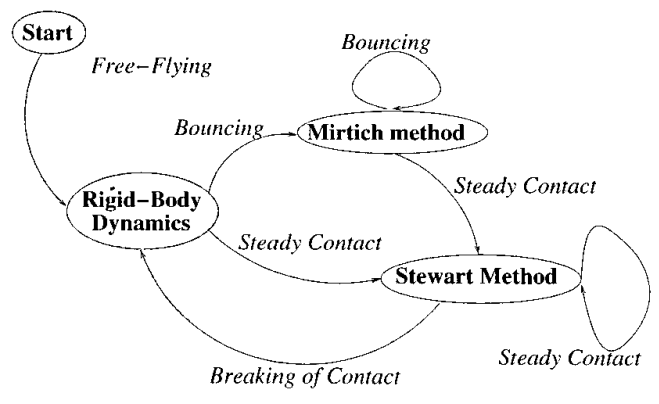

Figure 2: Control flow of adaptive contact simulation

1. Equation (8) means that the friction force is opposite to tangential velocity.

2. Equation (9) means that no contact means no contact forces.

3. Equation (10) means that if the tangential velocity is non-zero, then the friction force is maximized.

These equations are nonlinear, since $M, \mathbf{n}, D$, and $k$ all depend on $q(t)$ and $v(t)$.

However, if we let $k=k\left(q^{t}+h v^{t} / 2\right)$ and $M=$ $M\left(q^{t}+h v^{t}\right)$, we get a pure linear complementarity system as follows:

$$
\begin{aligned}
& {\left[\begin{array}{ccc}
D^{T} M^{-1} D & D^{T} M^{-1} \mathbf{n} & e \\
\mathbf{n}^{T} M^{-1} D & \mathbf{n}^{T} M^{-1} \mathbf{n} & 0 \\
-e^{T} & \mu & 0
\end{array}\right]\left[\begin{array}{c}
\beta \\
c_{n} \\
\lambda
\end{array}\right]+b=\left[\begin{array}{c}
\rho \\
\sigma \\
\zeta
\end{array}\right] \geq 0} \\
& \text { where } b=\left[\begin{array}{c}
D^{T}\left(v^{t}+h M^{-1} k\right) \\
\left(\mathbf{n}^{T} q^{t}-\alpha_{0}\right) / h+\mathbf{n}^{T}\left(v^{t}+h M^{-1} k\right) \\
0
\end{array}\right] \\
& \text { and }\left[\begin{array}{c}
\beta \\
c_{n} \\
\lambda
\end{array}\right] \geq 0,\left[\begin{array}{c}
\beta \\
c_{n} \\
\lambda
\end{array}\right]^{T}\left[\begin{array}{c}
\rho \\
\sigma \\
\zeta
\end{array}\right]=0
\end{aligned}
$$

This problem formulation conforms to the LCP as is given by Equation (5) and is solved in terms of the solution vector $z=\left[\begin{array}{lll}\beta^{T} & c_{n} \lambda\end{array}\right]$. This LCP is proven to have solution(s) which are unique for most problems although uniqueness is not guaranteed. However, Anitescue proved the existence of the solution(s) by a modification this LCP formulation [1].

\section{Implementation Details}

\subsection{Hybrid Scheme}

Our adaptive scheme for handling contact during simulation is represented in the state-transition diagram as shown in Figure 2. During simulation of rigid bodies, whenever a bouncing contact is detected, Mirtich's algorithm is applied, while Stewart's method is applied to cases of steady contact. A steady contact is detected when the bounces become so small that it is considered that the bouncing has ended. We use a tolerance to detect this during contact simulation. All these transitions in contact dynamics occur automatically during simulation using an adaptive scheme that keeps track of changes in the contact situation.

The simulation for contact situations is driven by contacts. This means that a 'Contact' class is instantiated at each time step whenever a collision is detected and an explicit (via Mirtich's algorithm) or implicit (via Stewart's LCP algorithm) calculation of impulse is performed using all the kinematics and dynamic information available within it. This approach provides scalability when it is applied to the multiple contact case. The formulation for computing contact dynamics is correspondingly scaled in a natural manner in proportion to the complexity of the number of contacts.

The LCP formulations were solved using a numerical library developed based on Lemke's algorithm which is a pivoting method similar to the simplex method for linear programming. Specifically, we have used the mathematical library, Meschach [10], which is written in C. We call Meschach's functions from IGMS.

\subsection{System Architecture}

Our adaptive scheme for dealing with contacts has been incorporated into our general-purpose dynamic simulator, I-GMS [7]. I-GMS is an object-oriented dynamic simulator which can simulate various types of bodies such as linkages and free bodies. Classical rigid body dynamics is used to simulate free bodies with contact dynamics applied to free rigid bodies whenever contact occurs. The adaptive contact-handling scheme was implemented as a method available with the object class, FreeBody, which represents the rigid bodies. (This class itself is derived from another class, Body, which represents more general types of bodies.) This addition of contact dynamics to the system architecture of the existing I-GMS is shown in the Figure 3.

\section{Simulation Example}

A three dimensional ball with an initial velocity is thrown onto a flat rigid surface and it starts to bounce, roll, slide, and eventually comes to a stop after a period of 'steady contact'. Here, we consider the contact in steady state once the bounces become very small on the flat surface. 


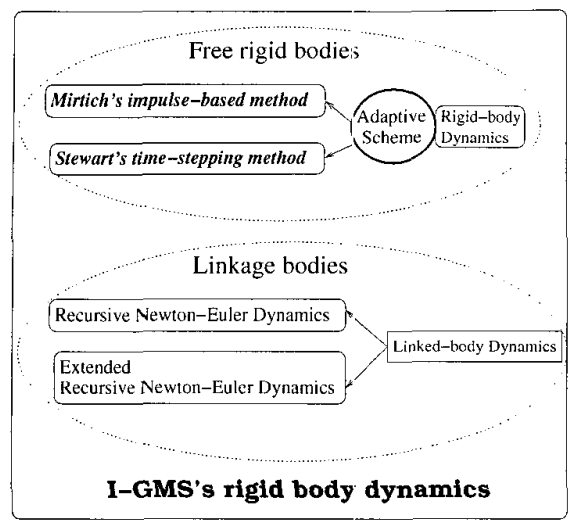

Figure 3: Incorporation of contact dynamics into IGMS

In our experiment, we have used various parameters to illustrate the change of the system's behavior. These are the coefficient of friction $(\mu)$ and the coefficient of restitution $(\varepsilon)$ which are used for Mirtich's algorithm. We have also attempted to vary the coefficient of friction in Stewart's algorithm. Recall that Stewart's method assumes zerc coefficient of restitution.

Note that in the plots (Figure 4) for the simulation results, we have shown only the $z$-direction position trajectories of the ball and the normal impulse trajectories to help illustrate the falling and bouncing situations of the ball in three dimensions. In Mirtich's method, the impulse calculated is the total impulse applied during compression and the restitution phases of the collision, whereas it is the integrals of the forces over each time step in Stewart's method.

In Figure 4(a), we show the results of applying Mirtich' algorithm. A ball is colliding with the flat rigid surface around time step 40 and starts to slide and roll. As the plots show, setting the coefficient of restitution close to ' 1 ' brings the system's state into an unstable one, making the ball bounce forever without coming to rest after an initial period of fluctuation. We expect the ball to bounce for some initial transient period until it rests in a steady state. Although we get more stable behavior as we decrease $\varepsilon$, the ball continues to bounce.

In our hybrid scheme (see Figure 4(b)), Mirtich's algorithm is applied when the ball first hits the surface and then Stewart's algorithm takes over the state evolution once bouncing becomes small enough to be regarded as 'steady contact'. This transition in contact dynamics happens automatically during simulation using our adaptive transition scheme which checks bouncing status in real-time. Figure 4(b) shows the change of contact status from 'bouncing' to 'steady' for several different $\varepsilon$ values, after going through an initial transient period of bouncing. The duration of this transient period depends on $\varepsilon$. Generally, lower $\varepsilon$ values produce smaller transient periods, which conforms to physical accuracy. Unlike Mirtich's algorithm, Stewart's algorithm assumes zero coefficient of restitution and this make it suited to dealing with 'steady contact' situations. Thus, it works well for the case when the ball comes to a rest, avoiding any possibility of an unstable system state as may occur in Mirtich's algorithm. Note that we have used a low coefficient of restitution to prolong the duration of the sliding and rolling motion of the ball on the surface, which is eventually stabilized by our hybrid scheme into a steady state.

\section{Discussion and Future Work}

We have developed a hybrid scheme to simulate contact with Coulumn friction of a system of rigid bodies, which has been incorporated into our generalized motion simulator, I-GMS. In particular, this scheme has an adaptive capability to change over to appropriate contact dynamics depending on two contact situations, 'bouncing' and 'steady'. Our hybrid scheme shows some potential for physically-correct simulation of rigid body contact, while maintaining real-time efficiency at the same time by using two impulse-based methods, Mirtich and Stewart's algorithms.

Still there are some stability issues remaining to be solved. Especially, dynamic simulation with contact involves fine step size tuning during simulation which requires applying an adaptive step size for correct simulation without numerical failure. Extending the formulation to linked-body contact problems would be another issue that should be dealt with, if the hybrid scheme will be applied to general simulation environments.

\section{References}

[1] M. Anitescu, F. A. Potra, and David Stewart. Timestepping for three-dimensional rigid body dynamics. Technical Report, April 1998.

[2] David Baraff. Dynamic simulation of nonpenetrating rigid bodies. In Ph.D Thesis, March 1992.

[3] P. Lötstedt. Numerical simulation of time-dependent contact friction problems in rigid body mechanics. Zeitschrift für Angewandte Mathematik and Mechanik, 5:370-393, 1984.

[4] Monteiro Marques. Differential inclusions in nonsmooth mechanical problems: Shocks and dry friction. In Progress in Nonlinear Differential Equations and Their Applications, volume 9, 1993. 

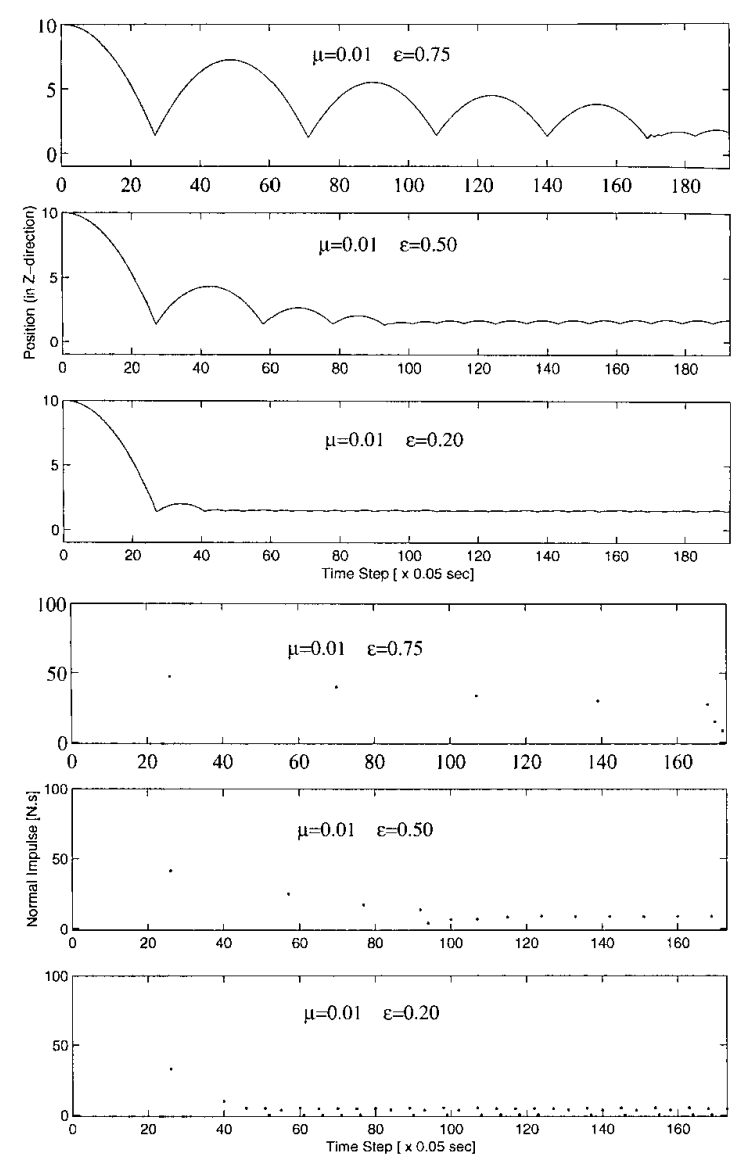

(a)
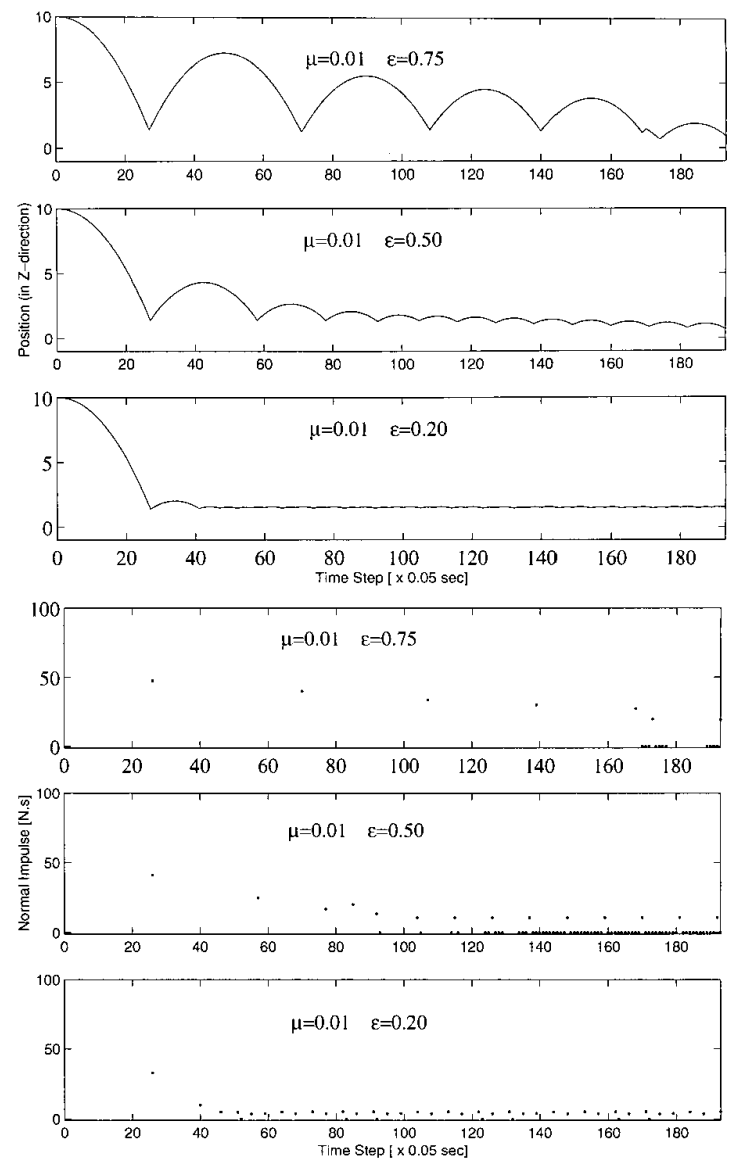

(b)

Figure 4: Impulse and position trajectories for the falling-ball simulation (a) Using Mirtich algorithm, (b) Using the hybrid algorithm.

[5] Brian Mirtich. Impulse-based dynamic simulation of rigid body systems. In Ph.D Thesis, Fall 1996.

[6] J.J. Moreau. Unilateral contact and dry friction in finite freedom dynamics. In Nonsmooth Mechanics and Applications, pages 1-82, 1988.

[7] Wookho Son, Kyunghwan Kim, and Nancy M. Amato. An interactive generalized motion simulator (I-GMS) in an object-oriented framework. In Proceedings of Computer Animation 2000, Philadelphia, Pennsylvania, May 2000.

[8] Wookho Son, Kyunghwan Kim, Nancy M. Amato, and Jeffrey C. Trinkle. Interactive dynamic simulation using haptic interaction. In Proc. IEEE Int. Conf. Intel. Rob. Syst. (IROS), Takamatsu, Japan, November 2000.

[9] Peng Song, Peter Kraus, and Vijay Kumar. Analysis of rigid body dynamic model for simulation of systems with frictional contacts. volume 4, pages 267-279, 1998.
[10] David E. Stewart and Z. Leyk. Meschach: Matrix computations in C. In Proceedings of the CMA, 32, Australian National University, 1994.

[11] David E. Stewart and J. C. Trinkle. An implicit timestepping scheme for rigid-body dynamics with inelastic collisions and coulomb friction. In International Journal of Numerical Methods for Engineering, volume 39, pages 2673-2691, 1996.

[12] David E. Stewart and J. C. Trinkle. An implicit time-stepping scheme for rigid-body dynamics with coulomb friction. In Proc. IEEE Int. Conf. Robot. Autom. (ICRA), pages 162-169, San Francisco, CA, 2000 .

[13] J. C. Trinkle, J. S. Pang, S. Sudarsky, and G. Lo. On dynamic multi-rigid-body contact problems with coulomb friction. Zeitschrift für Angewandte Mathematik and Mechanik, 4:267-279, 1997. 